\title{
Isolation, identification and toxinotyping of Clostridium perfringens isolated from broilers in Pakistan
}

\author{
Zain Ul Abadeen ${ }^{1}$, Muhammad Tariq Javed ${ }^{1, *}$, Farzana Rizvi ${ }^{1}$ and Sajjad Ur Rahman ${ }^{2}$ \\ ${ }^{1}$ Department of Pathology, Faculty of Veterinary Science, University of Agriculture, 38040 Faisalabad, Pakistan; ${ }^{2}$ Institute of \\ Microbiology, Faculty of Veterinary Science, University of Agriculture, 38040 Faisalabad, Pakistan \\ *Corresponding author's e-mail: mtjaved@uaf.edu.pk
}

\begin{abstract}
Necrotic enteritis (NE) is one of the important enteric disease in the poultry industry worldwide, caused by C. perfringens type A. This study describes the isolation, identification, and toxinotyping of $C$. perfringens in necrotic enteritis affected broiler chicken in Pakistan. A total of 430 intestinal samples from dead carcasses and birds suspected of NE outbreak, in and around Faisalabad, Pakistan were collected from 36 broiler farms which yielded 87 alpha toxin gene ( $c p a)$ positive $C$. perfringens type A isolates. The birds having 4-5 weeks of age, clinical signs, and reared in open (conventional) sheds showed higher $C$. perfringens isolation rate. The study concluded netB negative $C$. perfringens type $\mathrm{A}$ as a causative agent for NE outbreaks in broiler birds in Faisalabad, Pakistan.
\end{abstract}

Keywords: Clostridium perfringens, necrotic enteritis, toxinotyping, chicken, Pakistan.

\section{INTRODUCTION}

The poultry industry has experienced exponential growth since 1960s and has provided major contributions towards protein consumption in humans in Pakistan (Hussain et al., 2015). Pakistan is ranked as the $11^{\text {th }}$ largest poultry producing country in the world and this industry has grown at a rate of $8-10 \%$ annually and contributed $35 \%$ of the total meat produced in the country and created jobs for more than 1.5 million people. (Anonymous, 2019-20).

Despite rapid development in the industry, infectious diseases have been a threat at large to the farms. Major infectious diseases include Newcastle Disease (ND), colibacillosis, Infectious Coryza (IC), coccidiosis, Infectious Bronchitis (IB), enteritis, salmonellosis, fowl pox, avian influenza, and hydro-pericardium syndrome (Ahmad et al., 2008 and Mustafa and Ali, 2005). Environment factors, e.g., (litter quality, over-crowding, ventilation) and nutritional imbalances (high crude protein feed) may predispose the birds towards vulnerability. Nutritional factors might influence gut properties, gut flora, and immunity levels in the birds (Moore, 2016).

Necrotic enteritis (NE) is an important ailment in the poultry industry caused by Clostridium $(C$.) perfringens type A. It was first described by Perish in 1961 and has been classified into clinical and sub-clinical forms. The clinical form is associated with obvious signs, e.g., weight loss, ruffled feathers, high mortality, diphtheroid membrane, and necrotic foci in intestinal mucosa along with gas accumulation however, the sub-clinical form is associated with less prominent signs, i.e., poor feed conversion ratio, high morbidity, and low mortality. The antibiotic growth promoters (AGPs) have been used for the control and treatment of NE. Recently, AGPs have been banned in poultry with eventual re-emergence of NE in poultry (Immerseel $e t$ al., 2009).

C. perfringens is a gram-positive, spore forming, non-motile and anaerobic bacillus that resides in the gastrointestinal tract of humans and animals as part of normal gut flora. Based on the toxins produced, it is classified into five toxinotypes (A, $\mathrm{B}, \mathrm{C}, \mathrm{D}$, and $\mathrm{E}$. The main toxins produced are alpha, beta, epsilon, iota, and enterotoxin. A novel necrotic enteritis Blike (NetB) toxin (Net-B), is characterized as B-pore forming enterotoxin is also considered a virulence factor for avian $\mathrm{NE}$ (Keyburn et al., 2008). However, cases of NE have been identified in poultry by $C$. perfringens type A, which are negative for $n e t B$ gene indicating other virulence factors involved in the occurrence of NE. The $C$. perfringens producing alpha and NetB toxins are supposedly classified into type G (Smyth and Martin, 2010; Rood et al., 2018). From last few years, large number of NE outbreaks were being reported in and around the areas of Faisalabad reported by field veterinarians and various diagnostic labs including at Department of Pathology, University of Agriculture,

Abadeen, Z.U., M.T. Javed, F. Rizvi and S.U. Rahman.2021. Isolation, identification and toxinotyping of Clostridium perfringens isolated from broilers in Pakistan. Pak. J. Agri. Sci. 58:1367-1372.

[Received 22 Dec 2020; Accepted 11Jun 2021; Published (online) 21 Sep 2021]

(c) (1) 
Faisalabad, Pakistan by receiving birds for clinical and postmortem examination.

The current study was designed to update the NE status, isolate $C$. perfringens from suspected NE in broiler birds and characterize the isolates by amplification of toxin gene specific primers for precise identification and awareness of the infection in the study area.

\section{MATERIALS AND METHODS}

Collection and shipment of the samples: A total of 36 broiler farms were visited, which were suspected of NE outbreak, in and around Faisalabad, Pakistan during September 2017March 2019. A total of 430 intestinal samples were collected from diseased and suspected live birds based on clinical and sub-clinical signs for postmortem examination and sample collection. The clinical signs included poor body condition, ruffled feathers, diarrhea, dehydration, and weight loss. The gross lesions, e.g., necrotic foci and ballooning of the intestines were considered characteristic during postmortem examination. The severity of infection was characterized by a lesion scoring system as described earlier (Prescott et al., 1978). The jejunum of the small intestines along with Meckel's diverticulum was collected aseptically into sterile Phosphate-buffered Saline (PBS) and transported to Laboratory of Molecular Pathology, Department of Pathology, University of Agriculture, Faisalabad, Pakistan. The samples were stored at $4{ }^{\circ} \mathrm{C}$ until further processed.

Isolation and biochemical identification: The gut samples were enriched into a $5 \mathrm{ml}$ sterile Reinforced Clostridium Medium (RCM) (Oxoid, USA) at $37^{\circ} \mathrm{C}$ for $24-36$ hours under anaerobic conditions. The anaerobiosis was generated by using $\mathrm{CO}_{2}$ gas producing packs, i.e., AnaeroGen ${ }^{\circledR}$ (Oxoid, USA) in AnaeroJarTM (Oxoid, USA) considering $<1 \% \mathrm{O}_{2}$ levels, while achieving 9-13\% $\mathrm{CO}_{2}$ levels within 30 mins.
Later, a loopful from the broth was inoculated on 5\% Sheep Blood Agar (SBA) (Oxoid, USA), egg yolk emulsion (EYE) agar (Oxoid, USA), and Perfringens Tryptose Sulphite Cycloserine (PTSC) agar (Oxoid, USA) plates separately and incubated at $37{ }^{\circ} \mathrm{C}$ for 24-36 hours under anaerobic conditions as described previously. The double zoned $\beta$-hemolysis colonies on SBA, black colored colonies on PTSC agar, and opalescent growth on EYE agar plates were suspected as $C$. perfringens and were then, subjected for Gram's staining procedure and biochemical identification via Indole, VogesProskauer, carbohydrate fermentation, methyl red, and nitrate reduction tests (Miah et al., 2011). The colonies confirmed by biochemical tests criteria were subjected cultured further for obtaining pure growth on PTSC agar and finally stored at -20 ${ }^{\circ} \mathrm{C}$ in a sterile glycerol medium until further used.

Molecular identification: The DNA was extracted from pure colonies by boiling the colonies in $200-\mu \mathrm{l}$ sterile nucleases free water in an Eppendorf ${ }^{\circledR}$ tube $(500 \mu \mathrm{l})$ for 20 mins at 100 ${ }^{\circ} \mathrm{C}$ in a water bathtub (Songer and Meer, 1996). After centrifugation at $15,000 \mathrm{~g}$ for two mins, a $10-\mu 1$ supernatant was collected in a nucleases free collection tube and stored at $-20{ }^{\circ} \mathrm{C}$ until further use.

The molecular identification was done by species-specific $16 \mathrm{~S}$ rRNA primers in a conventional PCR method followed by visualization of the PCR products on $1.5 \%$ agarose gel under Gel Doc ${ }^{\circledR}$ EZ Imager (Bio-Rad, USA). For each reaction, $50 \mu 1$ of master mix containing $25 \mu 1$ Dream Taq Green PCR Master Mix (2X) (Thermo Scientific ${ }^{\circledR}$, USA), 5 $\mu 1$ template DNA, forward and reverse primers ( $2 \mu \mathrm{l}$ each $)$ and sterile nucleases free water $(18 \mu \mathrm{l})$ was taken and their mix transferred to PCR tubes. The PCR conditions were as follows: denaturation at $94{ }^{\circ} \mathrm{C}$ for $1 \mathrm{~min}$, annealing at $53{ }^{\circ} \mathrm{C}$ for $1 \mathrm{~min}$, and extension at $72^{\circ} \mathrm{C}$ for 1 min having 35 cycles each (Wang et al., 1994).

Table 1.Clostridium perfringens specific genes and primer sequences used for molecular identification and typing

\begin{tabular}{|c|c|c|c|}
\hline Gene detected & Primer sequence (5'-3') & Product size (bp) & Reference \\
\hline \multirow[t]{2}{*}{ 16S rRNA } & AAAGATGGCATCATCATTCAAC & 279 & (Wang et al., 1994) \\
\hline & TACCGTCATTATCTTCCCCAAA & & \\
\hline \multirow[t]{2}{*}{ cpa } & GTTGTAAGCGCAGGACATGTTAAG & 402 & (Yoo et al., 1997) \\
\hline & CATGTAGTCATCTGTTCCAGCATC & & \\
\hline \multirow[t]{2}{*}{$c p b$} & ACTAATACAGACAGATCATTCAACC & 236 & \\
\hline & TTAGGAGCAGTTAGAAACTACAGAC & & \\
\hline \multirow[t]{2}{*}{$e t x$} & ACTGCAACTACTACTCATACTGTG & 541 & \\
\hline & CTGGTGCCTTAATAGAAAGACTCC & & \\
\hline \multirow[t]{2}{*}{ cpi } & GCGATGAAAAGCCTACACCACTAC & 317 & \\
\hline & GGTATATCCTCCACGCATATAGTC & & \\
\hline \multirow[t]{2}{*}{$c p b 2$} & GAAAGGTAATGGAGAATTATCTTAATGC & 573 & (Herholz et al., 1999) \\
\hline & GCAGAATCAGGATTTTGACCATATACC & & \\
\hline \multirow[t]{2}{*}{ cpe } & GGAGATGGTTGGATATTAGG & 233 & (Meer and Songer, \\
\hline & GACAGGGGCATACCCATATA & & 1997) \\
\hline \multirow[t]{2}{*}{ netB } & GCTGGTGCTGGAATAAATGC & 383 & (Keyburn et al., 2010) \\
\hline & TCGCCATTGAGTAGTTTCCC & & \\
\hline
\end{tabular}


Toxinotyping: The identification of the virulence genes for various toxins i.e., alpha (cpa), beta (cpb), beta $2(c p b 2)$, epsilon (etx), iota (cpi), enterotoxin (cpe) and NetB (netB) was done by using PCR thermocycler Qantarus ${ }^{\circledR}$ Q-Cycler (Quanta Biotech, England) (Table 1). For $c p a, c p b$, etx, and $c p i$, the PCR conditions were used as: initial denaturation at $95{ }^{\circ} \mathrm{C}$ for 5 mins followed by denaturation at $94^{\circ} \mathrm{C}$ for $1 \mathrm{~min}$, annealing: $55^{\circ} \mathrm{C}$ for $1 \mathrm{~min}$ and extension at $72{ }^{\circ} \mathrm{C}$ for $1 \mathrm{~min}$; 30 cycles each and final extension at $72{ }^{\circ} \mathrm{C}$ for 3 mins (Yoo et al., 1997). For $c p b 2$, initial denaturation at $95^{\circ} \mathrm{C}$ for 2 mins, denaturation at $94{ }^{\circ} \mathrm{C}$ for 30 secs, annealing at $48{ }^{\circ} \mathrm{C}$ for 30 secs, extension at $72{ }^{\circ} \mathrm{C}$ for 30 secs; 35 cycles each and final extension at $72{ }^{\circ} \mathrm{C}$ for 10 mins (Herholz et al., 1999). The PCR conditions for cpe included initial denaturation at $94{ }^{\circ} \mathrm{C}$ for 4 mins, denaturation at $94^{\circ} \mathrm{C}$ for $1 \mathrm{~min}$, annealing at $53^{\circ} \mathrm{C}$ for $1 \mathrm{~min}$, extension at $72^{\circ} \mathrm{C}$ for $1 \mathrm{~min} ; 35$ cycles each and final extension at $72{ }^{\circ} \mathrm{C}$ for $10 \mathrm{~min}$ (Meer and Songer, 1997). For netB, denaturation at $94{ }^{\circ} \mathrm{C}$ for 30 secs, annealing at 55 ${ }^{\circ} \mathrm{C}$ for 30 secs and extension at $72{ }^{\circ} \mathrm{C}$ for 1 min having 35 cycles each (Keyburn et al., 2010). All PCR products were
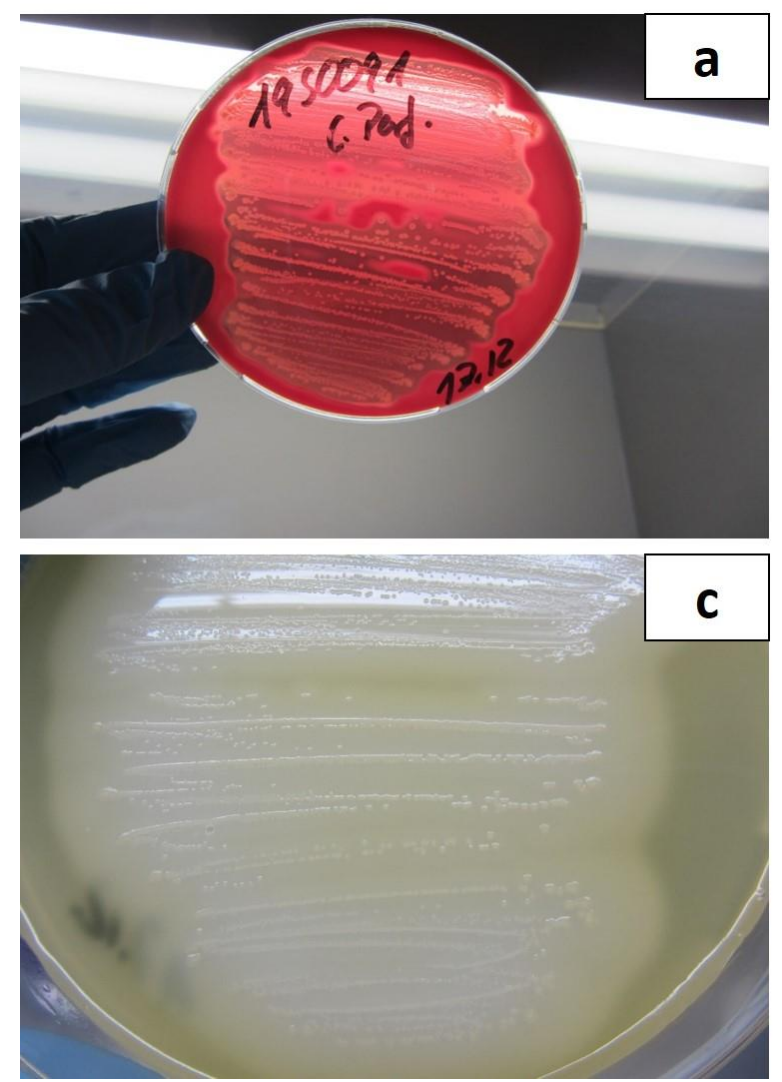

visualized by $1.5 \%$ agarose Gel Electrophoresis method under Gel Doc ${ }^{\circledR}$ EZ Imager (Bio-Rad, USA).

Statistical analysis: The proportion of culture positive for Clostridium was calculated by dividing the number of positive culture samples divided by the total number of samples analyzed. Furthermore, its confidence interval (CI) was estimated by the exact $95 \%$-Clopper \& Pearson interval method. Fisher's Exact test was used to perform the univariable analysis, considering the level of significance as $\mathrm{p}<0.05$. The dependent variable was culture positive outcome, and explanatory variables were obtained from a questionnaire filled during sampling. The statistical analyses for the current findings were performed using $\mathrm{R}$ (http://www.Rproject.org/), and R-Studio (RStudio).

\section{RESULTS}

A total of 87 isolates of $C$. perfringens were obtained through SBA, EYE, and PTSC culture media. C. perfringens showed double $\beta$-hemolysis on SBA (Fig. 1a), black colored colonies on PTSC agar (Fig. 1b), and opalescent growth on EYE agar
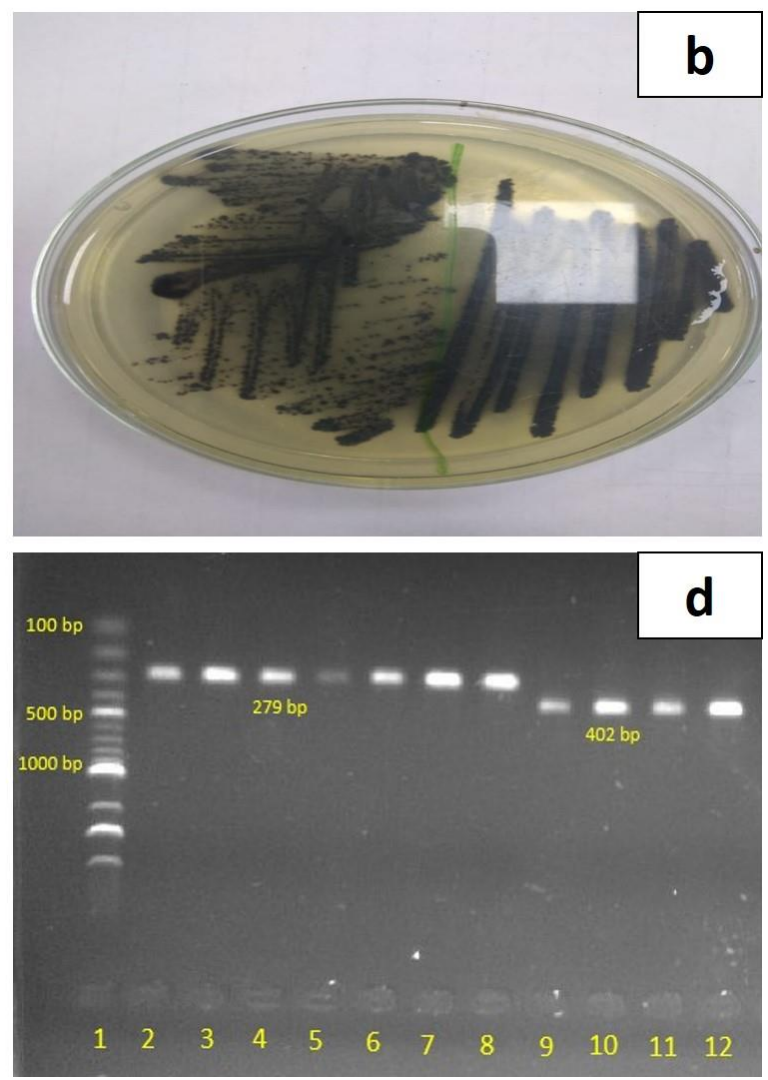

Figure 1. a) Presence of double zone of hemolysis produced by $C$. perfringens on SBA (b) Characteristic black colored colonies of $C$. perfringens on PTSC agar (c) Presence of opalescent growth produced by $C$. perfringens on EYE agar (d) Photograph of a PCR gel showing $C$. perfringens isolates harboring 16S rRNA and alpha gene where, lane 1: 100 bp ladder, lane 2-8: isolates carrying 16S rRNA (279 bp) and lane 9-12: isolates carrying alpha gene (402 bp). 
Abadeen, Javed, Rizvi \& Rahman

Table 2. List of tests performed for the identification of $C$. perfringens.

\begin{tabular}{cc}
\hline Test performed & Result \\
\hline Perfringens Tryptose Sulphite Cycloserine (PTSC) agar & Black colored colonies \\
$5 \%$ Sheep blood agar (SBA) & Beta hemolysis (double zone of hemolysis) \\
Egg yolk emulsion (EYE) agar & Opalescent growth (lecithinase activity) \\
Gram's staining & Gram-positive, rod-shaped bacilli \\
Carbohydrate fermentation & +ve and produced gas \\
Methyl Red & +ve \\
Nitrate reduction & $+\mathrm{ve}$ \\
Voges-Proskauer & -ve \\
Indole & -ve \\
\hline
\end{tabular}

Table 3. Univariable analysis $(n=430)$

\begin{tabular}{lllll}
\hline Variables & Category & Positive \% & OR (95\% CI) & p-value* \\
\hline Age (weeks) & $<2-3(\mathrm{n}=29)$ & $3.4 \%(1 / 29)$ & Ref & $<0.001$ \\
& $>3-4(\mathrm{n}=56)$ & $5.4 \%(3 / 56)$ & $1.58(0.16-15.95)$ & \\
& $>4-5(\mathrm{n}=262)$ & $26.3 \%(69 / 262)$ & $10.01(1.34-74.98)$ & \\
Infection & $>5-6(\mathrm{n}=83)$ & $16.9 \%(14 / 83)$ & $5.68(0.71-45.28)$ & \\
Severity & Subclinical $(\mathrm{n}=298)$ & $3.7 \%(11 / 298)$ & Ref & $<0.001$ \\
Farming type & Clinical $(\mathrm{n}=132)$ & $57.6 \%(76 / 132)$ & $35.41(17.69-70.88)$ & \\
& Environment-controlled $(\mathrm{n}=246)$ & $9.8 \%(24 / 246)$ & Ref & $<0.001$ \\
& Open $(\mathrm{n}=184)$ & $34.2 \%(63 / 184)$ & $4.79(2.79-8.46)$ & \\
\hline
\end{tabular}

*p-value <0.05 considered as significant; temperature, feeding, drinking, and the environment were controlled automatically; open (conventional) sheds where temperature, feeding, watering and ventilation were controlled manually.

plates (Fig. 1c). The colonies of these isolates appeared as Gram-positive rods on staining, negative by indole and Voges-Proskauer tests and positive by Methyl red, carbohydrate fermentation and nitrate reduction tests (Table 2). By PCR, 87/87(100\%) of suspected amplified the DNA for $C$. perfringens species-specific 16S rRNA PCR. These isolates did not amplify $c p b, c p b 2$, etx, cpi, cpe, and $n e t B$ genes but did amplify cpa gene only. Hence, all isolates were considered as $C$. perfringens type A (Fig.1d).

Variables, e.g., age, infection severity, and type of farming showed the highest number of confirmed positive cases were from flocks having 4-5 weeks of age, i.e., 26.3\% (69/262) compared to $10.7 \%(18 / 168)$ in all other age groups. The PCR results related to the severity of the infection showed that the presence of $C$. perfringens was largely confirmed in clinical cases $57.6 \%$ (76/132) compared to subclinical cases, i.e., $3.7 \%$ (11/298). The C. perfringens was found to be highly isolated from samples collected from open (conventional) sheds $72.4 \%(63 / 87)$ as compared to environment-controlled sheds, i.e., $27.6 \%$ (24/87). Overall, $20.2 \%$ ( 87 out of $430,95 \%$ CI: $16.53-24.34 \%$ ) samples were positive for $C$. perfringens (Table 3).

\section{DISCUSSION}

Necrotic enteritis (NE) is an important poultry disease and the losses estimated due to NE in the poultry industry ranges between 2 to 6 billion US dollars, globally (Mwangi et al., 2019). Previously, C. perfringens has been isolated from poultry fecal material and meat products obtained from commercial poultry and small ruminants in Pakistan (Khan et al., 2015; Achakzai et al., 2020; Khan et al., 2020 and Khan et al., 2021). It has potential public health importance. We used culture examination, biochemical tests, and molecular based identification techniques to detect and identify $C$. perfringens in our suspected samples. Later, these isolates were differentiated based on 16S rRNA for species identification and the presence of toxin genes.

The $C$. perfringens type A has been associated with cpa gene for the production of $\alpha$ toxin and is considered important in the etiology for NE in the poultry. In the present study, all isolates were positive for only cpa gene and grouped as toxinotypes A. These results are in line with the findings of Khan et al. (2021) found both types A and G of C. perfringens from fecal samples of commercial broilers from Pakistan. Another important toxin the NetB, is a pore-forming toxin that has been described as one of the main virulence factors for NE outbreaks in birds (Keyburn et al., 2008). The present study indicated all $C$. perfringens isolates to be negative for $n e t B$ gene. Similar results have been reported in India, Iran, and Algeria (Datta et al., 2014; Merati et al., 2017; Razmyar et al., 2018). The lower levels of maternal antibodies up to 3 weeks of age in birds' circulatory system can induce a higher risk of NE. One of the key factors could be the stress caused by the alterations in the gastrointestinal flora by shifting feed from starter to grower. It might favor the environment for $C$. perfringens to proliferate (Moore, 2016). In the recent past, the subclinical form of NE has been reported more often than 
the clinical form. The sub-clinical form remains difficult to diagnose and the birds may remain undetected (Timbermont et al., 2011).

In our study, the positive percentage of $C$. perfringens at open (conventional) and environment-controlled sheds was up to $34.2 \%$ (63/184) and 9.8\% (24/246), respectively. In some countries e.g., Egypt, China, and Jordan, the prevalence of $C$. perfringens in live poultry was reported at $41 \%, 23.1 \%$, and 43.2\%, respectively (Gharaibeh et al., 2010; Osman et al., 2012; Zhang et al., 2018). The health of the broiler birds is associated with several management related key factors, e.g., ventilation, temperature, air, humidity, and litter quality. Normally, open (conventional) poultry sheds are economical and have the potential to be used under hot climate, but risk animal stress and pathogen infiltration in poultry birds. Any environmental stress may directly affect the intestinal physiology, health, and welfare of the birds and hence can increase the susceptibility to NE due to immuno-suppression (Tsiouris et al., 2015). The contamination of the farm environment may play an important role in colonization and toxinogenesis of $C$. perfringens in normal intestinal microflora, therefore, virulent strains may replace normal inhabitant strains of $C$. perfringens (Barbara et al., 2008). Farm biosecurity, in this scenario, is important to reduce the infection threat.

Conclusion: We concluded that $C$. perfringens type A is prevalent in the suspected clinical NE outbreaks in poultry in Faisalabad, Pakistan. The molecular analysis indicated that all C. perfringens isolates were having cpa gene only and were negative for $n e t B$ suggested the possibility of other factors involved in the pathogenesis of NE. The present study thus indicated that 4-5 weeks of age and the clinical NE as the most yielding form for $C$. perfringens in broiler birds.

Ethical statement: The study was conducted following the guidelines of "Biosafety committee and Punjab Biosafety rules-2014" as the study was approved by "Institutional Biosafety/Bioethics Committee" of the University of Agriculture, Faisalabad, Pakistan (vide letter No. 6560/ORIC; dated: 13.09.2017).

Acknowledgments: Dr. Christian Seyboldt, Prof. Heinrich Neubauer and Dr. Tariq Jamil at Institute for Bacterial Infections and Zoonoses (IBIZ), Friedrich-Loeffler-Institute (FLI), Jena, Germany and Dr. Khushal Khan Kasi at Institute of Epidemiology, FLI, Greifswald-InselRiems, Germany are highly acknowledged for their technical guidance. "Higher Education Commission (HEC)", Islamabad, Pakistan is highly thanked for funding the stay of Z.U.A. at Bacterial infections and Zoonoses (IBIZ), Friedrich-Loeffler-Institut (FLI), Germany under the program "International Research Support Initiative Program (IRSIP)”.

\section{REFERENCES}

Achakzai, R., M.K. Taj and K.B. Achakzai. 2020. Microbiological studies on Clostridium perfringens isolated from commercial poultry of Balochistan. Asian J. Biol. Life Sci. 9:204-208.

Ahmad, A., A. Hanif, A. Shahid, M. Najeeb and M. Ahmad. 2008. Study of disease outbreak in layer flocks in and around Sammundri area. Pak. J. Life Social Sci. 6:59-62.

Anonymous. 2019-20. Pakistan Economic Survey 2019-20. Finance and Economic Affairs Division, Ministry of Finance, Govt. of Pakistan, Islamabad. Pakistan.

Barbara, A.J., H.T. Trinh, R.D. Glock and J.G. Songer. 2008. Necrotic enteritis-producing strains of Clostridium perfringens displace non-necrotic enteritis strains from the gut of chicks. Veterinary Microbiol. 126:377-82.

Datta, S., N. Rakha, G. Narang, D. Arora and N. Mahajan. 2014. Prevalnce of $\alpha, \beta$ and NetB toxin producing strains of Clostridium perfringens in broiler chickens in Haryana. Haryana Vet. 53:39-42.

Gharaibeh, S., R. Al-Rifai and A. Al-Majali. 2010. Molecular typing and antimicrobial susceptibility of Clostridium perfringens from broiler chickens. Anaerobe 16:586-9.

Herholz, C., R. Miserez, J. Nicolet, J. Frey, M. Popoff, M. Gibert, H. Gerber and R. Straub. 1999. Prevalence of $\beta 2$ toxigenic Clostridium perfringens in horses with intestinal disorders. J. Clin. Microbiol. 37:358-361.

Hussain, J., I. Rabbani, S. Aslam, and H.A. Ahmad. 2015. An overview of poultry industry in Pakistan. Worlds Poult. Sci. J. 71:689-700.

Immerseel, F.V., J.I. Rood, R.J. Moore and R.W. Titball. 2009. Rethinking our understanding of the pathogenesis of necrotic enteritis in chickens. Trends Microbiol. 17:32-6.

Keyburn, A.L., J.D. Boyce, P. Vaz, T.L. Bannam, M.E. Ford, D. Parker, A.D. Rubbo, J.I. Rood and R.J. Moore. 2008. NetB, a new toxin that is associated with avian necrotic enteritis caused by Clostridium perfringens. PLoS Pathog. 4:1-11.

Keyburn, A.L., X.X. Yan, T.L. Bannam, F.V. Immerseel, J.I. Rood and R.J. Moore. 2010. Association between avian necrotic enteritis and Clostridium perfringens strains expressing NetB toxin. Vet. Res. 41:1-8.

Khan, M., J. Nazir, A.A. Anjum, M.U.D. Ahmad, M. Nawaz and M.Z. Shabbir. 2015. Toxinotyping and antimicrobial susceptibility of enterotoxigenic Clostridium perfringens isolates from mutton, beef and chicken meat. J. Food Sci. Technol. 52:5323-5328.

Khan, M.A., A.Z. Durrani, S.B. Khan, N.U. Khan, M.A. Khan, K. Prince, M. Ali, G. Rashid and A.U. Khan. 2020. Biomarkers for pathogenic Clostridium perfringens in small ruminants of Khyber Pakhtunkhwa, Pakistan. Pak. J. Zool. 52:107-114. 
Khan, M.U.Z., B. Liu, S. Yang, X. Xu, Y. Wang and J. Cai. 2021. Genetic diversity of Clostridium perfringens strains isolated from broiler chickens revealed by PFGE analysis in China and Pakistan. Pak. Vet. J. 41:85-91.

Meer, R.R. and J.G. Songer. 1997. Multiplex polymerase chain reaction assay for genotyping Clostridium perfringens. Am. J. Vet. Res. 58:702-705.

Merati, R., S. Temim and A.A.A.F. Mohamed. 2017. Identification and characterization of Clostridium perfringens isolated from necrotic enteritis in broiler chickens in Tiaret, Western Algeria. J. Faculty Vet. Med. Kafkas Uni. 23:595-601.

Miah, M., M. Asaduzzaman, M. Sufian and M. Hossain. 2011. Isolation of Clostridium perfringens, Causal agents of necrotic enteritis in chickens. J. Bang. Agricul. Uni. 9:97-102.

Moore, R.J. 2016. Necrotic enteritis predisposing factors in broiler chickens. Avian Pathol. 45:275-81.

Mustafa, M.Y. and S.S. Ali. 2005. Prevalence of infectious diseases in local and fayoumi breeds of rural poultry (Gallus domesticus). Punjab Univ. J. Zool. 20:177-80.

Mwangi, S., J. Timmons, S. Fitz-Coy and S. Parveen. 2019. Characterization of Clostridium perfringens recovered from broiler chicken affected by necrotic enteritis. Poult. Sci. 981:128-35.

Osman, K.M., Y.A. Soliman, Z.M.S. Amin and M.A.K. Aly. 2012. Prevalence of Clostridium perfringens type A isolates in commercial broiler chickens and parent broiler breeder hens in Egypt. Rev. Sci. Tech. 31:931-41.

Prescott, J.F., R. Sivendra and D.A. Barnum. 1978. The use of bacitracin in the prevention and treatment of experimentally-induced necrotic enteritis in the chicken. Can. Vet. J. 19:181-187.

Razmyar, J., M. Rezaee, A.R. Movassaghi and B. Shojadust. 2018. NetB negative Clostridium perfringens infection associated with acute necrotic enteritis in mynah (Acridotheres tristis), grey partridge (Perdix perdix) and turkey (Meleagris gallopavo). Iran. J. Vet. Sci. Technol. 9:64-69.

Rehman, H.U., N. Fawad, G. Abbas, G. Naheed, B. Siddique, F. Afzal, J. Munawar, M. Aurangzeb, M. Atif, I.U. Haq, J. Shafi, M.S. Farooq, Z.A. Tahir, M. Qamar, A. Hussain, M. Saleem, M.T. Saleem and M. Javed. 2013.
Surveillance of poultry diseases in Punjab province, Pakistan; Special reference to Newcastle disease. Res. J. Vet. Prac. 1:1-4.

Rood, J.I., V. Adams, J. Lacey, D. Lyras, B.A. Mcclane, S.B. Melville, R.J. Moore, M.R. Popoff, M.R. Sarker, J.G. Songer, F.A. Uzal and F.V. Immerseel. 2018. Expansion of the Clostridium perfringens toxin-based typing scheme. Anaerobe 53:5-10.

RStudio. An Integrated development environment for $\mathrm{R}$ software environment for statistical computing and graphic. Version 1.0.44-(C) 2009-2016 ed. Northern Ave, Boston, MA 02210, USA: RStudio, Inc. 250.

Smyth, J.A. and Martin, T.G. 2010. Disease producing capability of netB positive isolates of $C$. perfringens recovered from normal chickens and a cow, and net $B$ positive and negative isolates from chickens with necrotic enteritis. Vet. Microbiol. 146:76-84.

Songer, J.G. and Meer, R.R. 1996. Genotyping of Clostridium perfringens by polymerase chain reaction is a useful adjunct to diagnosis of clostridial enteric disease in animals. Anaerobe 2:197-203.

Timbermont, L., F. Haesebrouck, R. Ducatelle and F.V. Immerseel. 2011. Necrotic enteritis in broilers: an updated review on the pathogenesis. Avian Pathol. 40:341-7.

Tsiouris, V., I. Georgopoulou, C. Batzios, N. Pappaioannou, R. Ducatelle and P. Fortomaris. 2015. The effect of cold stress on the pathogenesis of necrotic enteritis in broiler chicks. Avian Pathol. 44:430-5.

Wang, R.F., W.W. Cao, W. Franklin, W. Campbell and C.E. Cerniglia. 1994. A $16 S$ rDNA-based PCR method for rapid and specific detection of Clostridium perfringens in food. Mol. Cell. Probes 8:131-137.

Yoo, H.S., S.U. Lee, K.Y. Park and Y.H. Park. 1997. Molecular typing and epidemiological survey of prevalence of Clostridium perfringens types by multiplex PCR. J. Clin. Microbiol. 35:228-32.

Zhang, T.F., W.T. Zhang, D.Y. Ai, R.R. Zhang, Q. Lu, Q.P. Luo and H.B. Shao. 2018. Prevalence and characterization of Clostridium perfringens in broiler chickens and retail chicken meat in central China. Anaerobe 54:100-3. 